\title{
APPLICATION FOR AN EARLY DETECTION OF TRANSMISSION PARAMETERS DEGRADATION OF POINT-TO-POINT MICROWAVE LINKS
}

\author{
Milan Bubniak \\ Bachelor Degree Programme (3), FEEC BUT \\ E-mail: xbubni03@stud.feec.vutbr.cz \\ Supervised by: Petr Musil \\ E-mail: xmusil56@stud.feec.vutbr.cz
}

\begin{abstract}
Radio equipment of microwave links operating in an outdoor environment under the influence of weather conditions is at risk of a link failure or device defect occurrence, as well as their indoor units. This paper aims to present the creation of a network monitoring application capable of timely detection of potential failures, based on analyzing trends in monitored device parameters. The architecture is being described, as well as a graphical user interface. Several failure detection methods are covered. Conslusion summarizes the development results and outlines a future of the application.
\end{abstract}

Keywords: microwave link, radio-relay, wireless, point-to-point, network monitoring, SLA, NOC, SNMP, .NET, WPF, InfluxDB, SQLite, OpenWeatherMap

\section{INTRODUCTION}

Microwave link is a widely used technology of long-range data transmission used in B2B as well as B2C telecommunication sectors. They are utilized by service providers either as last miles or in backhaul portion of the network [1, 2]. Customer contracts often include SLA (service-level agreement) specifying strict levels of link availability, packet loss, latency, jitter etc. [3]. Outages threaten the accomplishment of these levels on given data link which can be caused by defects of microwave units. This can be avoided, apart from various levels of redundancy, by effective monitoring of links.

This article deals with the creation of a monitoring tool in form of desktop application designed for specific characteristics of microwave links, periodically requesting status parameters of monitored devices by Simple Network Management Protocol. The application then analyzes gathered data and detects anomalies with several methods. A completed application should be ready for deployment in network operations centres of service providers, where it can improve reliability of their services in combination with timely replacement of the defective part of the link within preventive maintenance.

\section{APPLICATION DESIGN}

The application is designed as a compact executable, integrating the core of the application as well as a graphical user interface. It is assumed it will be run by small and medium service providers on a machine directly connected into the management sector of their network. The operator can access this machine directly or by remote desktop (e.g. in case of virtualized environment). Optionally with the use of VPN, the application can be run on any appropriate machine. Application core consists of components forming the SNMP manager, which gathers data from remote SNMP agents on monitored devices. Further components provide saving and reading data from the database environment, managing of the device definitions and analyzing of gathered data. Notification in an alarm form with various priority is created in case of positive anomaly detection. Graphical interface renders gathered data in realtime charts and shows lists of current and historical alarms. 


\section{APPLICATION DEVELOPMENT}

.NET Framework is used as application basis with code written in C\# language. The graphic interface utilizes WPF libraries and scheme. InfluxDB is chosen as a specialized NoSQL database for storing of gathered time series data due to its efficient compression and performance during their processing. [4]. Device and link definitions are stored in a compact SQLite database file of which database engine is embedded into the application in the form of a library. The application uses weather data provided by OpenWeatherMap API.

\subsection{CORE STRUCTURE}

Fig. 1 shows a diagram of key components of application core with their relations.

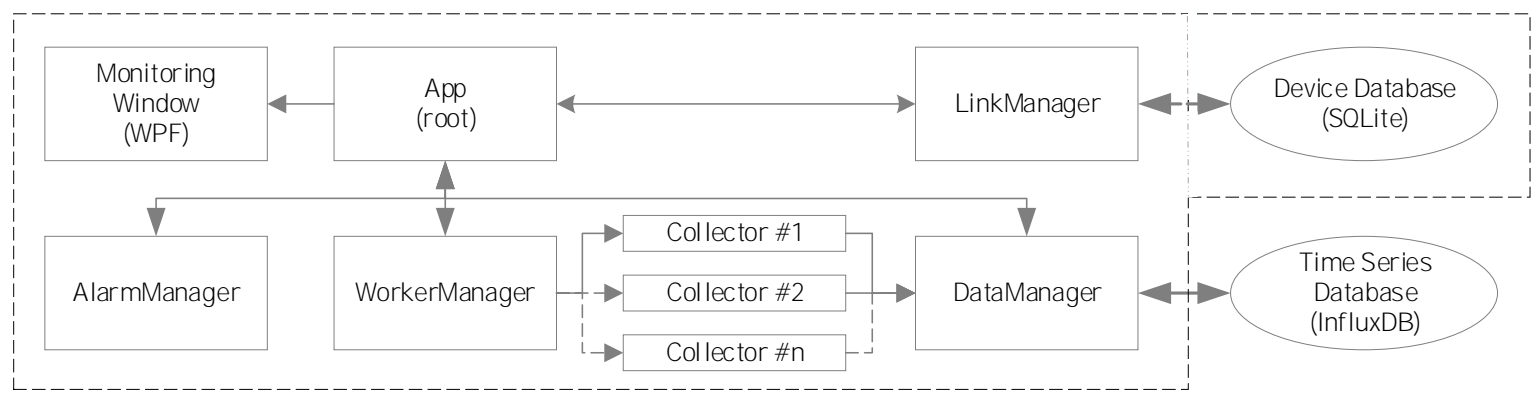

Figure 1: Diagram of application core components.

App - root object of a WPF application. Encapsulates Manager objects and WPF children objects. LinkManager - ensures reading and writing data to local SQLite database files. These include Device table with device definitions and monitoring configuration and Link table with an assignment of devices into link groups.

DataManager - provides caching of data from collectors which are intended to write into database and sends them periodically via HTTP REST API to InfluxDB server. Also contains reading methods. WorkerManager - initializes, starts and stops data collectors.

AlarmManager - periodically analyzes gathered data with several methods, contains device down and thresholds triggers, creates alarms when anomalies are detected and managing their collections.

Collectors - periodically collect values of monitored parameters from devices. Three types are currently implemented: SNMP collector sending PDU GetRequest with specific OID; Ping collector sending ICMP datagrams Echo Request; and Weather collector, sending HTTP requests to OpenWeatherMap API with geografical coordinates of a given device.

The application is built on asynchronous architecture because Collectors are highly input-output bound while Alarmmanager is I/O bound as well and also slightly CPU bound. Waiting for an answer from a microwave unit to SNMP PDU GetRequest can take up to several hundreds of milliseconds, or it may not arrive at all (SNMP PDUs are encapsulated in UDP datagrams on transport layer). Therefore collectors must be run in separate threads or called via Task $<\mathrm{T}>$ objects.

\subsection{USER INTERFACE STRUCTURE}

Fig. 2 shows the element layout of the application's main window in the current development version. In the left part is located links list with a search box. The bottom part is also divided into two parts, on the left side notification area is located which lists information, alarm creation events, warnings and errors, while on the right alarm lists are situated. Alarms can be acknowledged and moved into the appropriate tab. Each alarm row consists of creation time, measure name, link name, etc. 


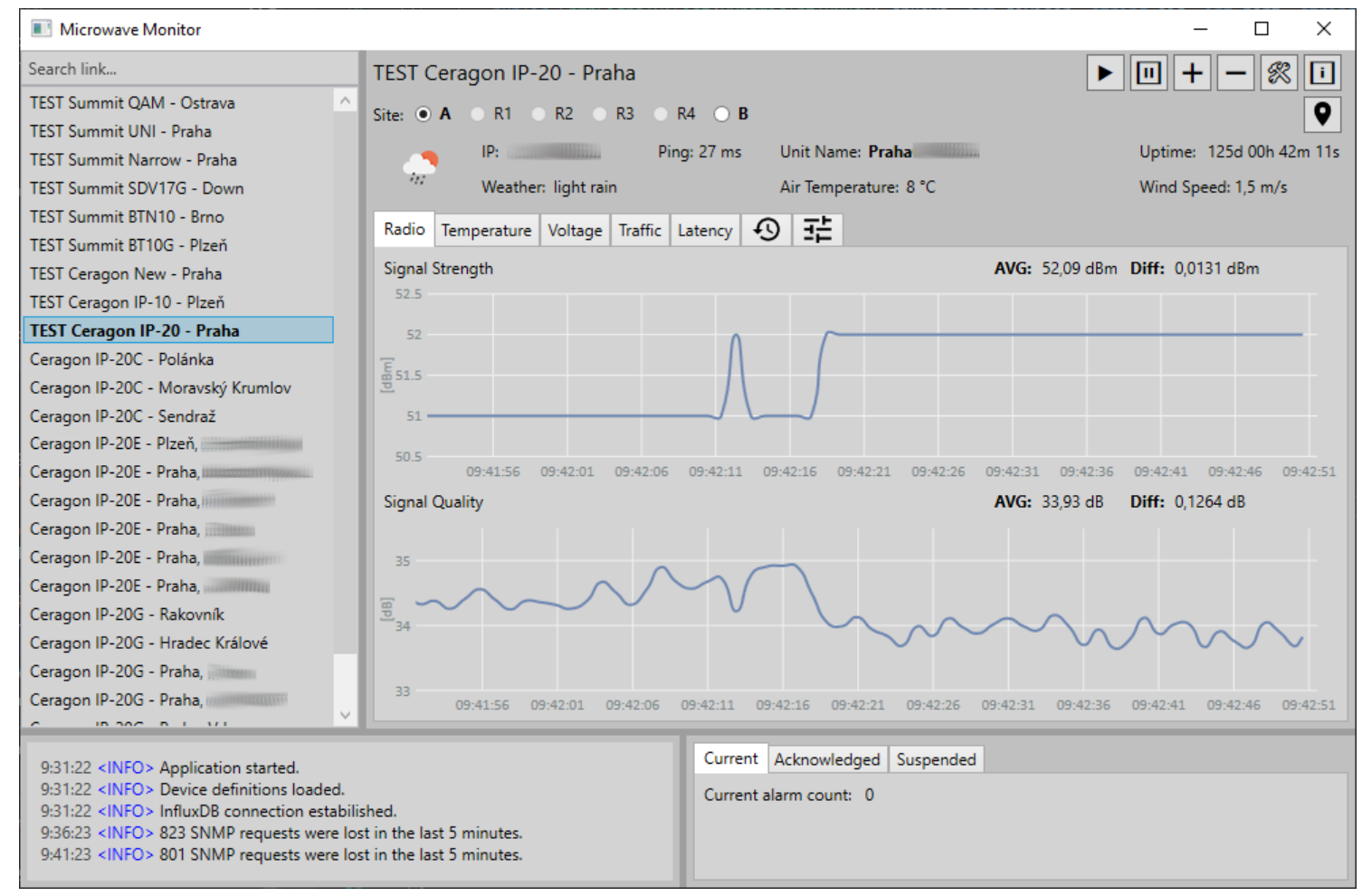

Figure 2: Screenshot of the running application (sensitive details are hidden).

A major part of main window area is populated by monitoring sector of selected link, with the header containing link name, site/device selection (up to six sites on a single link is supported), IP address of a selected device, control buttons and current site weather information. Parameters monitoring is divided into the tabs depending on the parameter type. Gathered values are displayed graphically in charts with realtime refreshing. Refresh interval is configurable for each parameter and device. In the current configuration, it is one second for most of the parameters. An important tab is Link Settings where the link monitoring configuration is being set. Also, the tab for historical reporting of parameters on the selected time window is included, but this function is not implemented yet.

\section{DATA ANALYZING METHODS}

Monitoring of the deviation from the long-term average performs calculation of long-term mean from values which age corresponds with the set time interval. The deviation is calculated as a difference of the long-term mean and a short-term mean. The latter is computed of values from the upper limit of set time interval until the present moment. Currently, two analyzes of this type are tested. The first one has a time interval set to values up to the weekly age with the upper age limit of 30 minutes. The second one has a time interval set to values up to the age of 60 minutes, with the upper limit of one minute. The maximum percentage of deviation at which an alarm is generated is user-configurable in the application settings. In the default configuration, it is $10 \%$ for the first analysis and $15 \%$ for the second analysis. Deviations are recalculated with each newly obtained value.

Long-term analysis by time serves as a backup method in case of a long-term decline in the trend of any of the parameters, which the monitoring of the deviation from the average is not able to detect. This method compares the current value of a parameter with historical values in the past days at the same time. An alarm is generated when the user-configurable percentage deviation is exceeded. Currently, a comparison of the last read value with the corresponding values up to the age of the one week is being tested at an interval of one minute. The default maximum deviation is $10 \%$. 
Analysis of parameter periodicity is an extension of the method of deviation from the long-term average. If any deviation exceeds the configurable percentage difference ( $3 \%$ by default), the timestamp of this value is stored and then compared with the last stamps recorded in this way. If the time gaps between them have constant intervals (with a tolerance of $1 \%$ of the time interval, but at least $\pm 1 \mathrm{~s}$ ), which may indicate continuous external interference for example, an alarm is generated.

Temperature correlation analysis is one of the next methods suitable for future implementation. It compares the air temperature obtained using the Weather API with the temperature of radio of the outdoor microwave unit (ODU) considering current weather state and wind speed. Fig. 3 shows a sample of a linear relation between these temperatures. Safe temperature range will be determined for each type of ODU. Deviations from this range will then be considered as an impending radio failure.

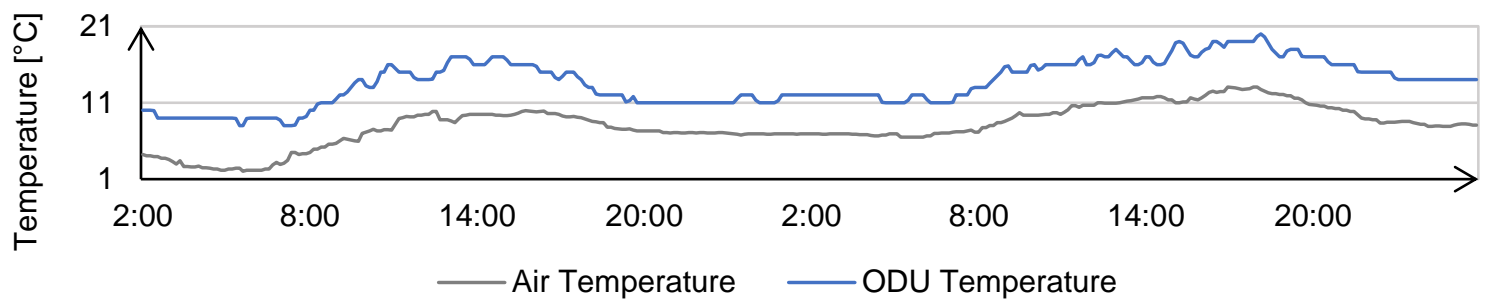

Figure 3: Sample of the linear relation between ODU temperature and outdoor ambient temperature. Data were obtained via the application during its continuous run.

\section{CONCLUSION}

The created application, although still in the development stage, can read parameters from remote devices using defined SNMP OIDs, measure latency and download weather information for each individual site. It performs effective monitoring, with the added value of user comfort thanks to an environment tailored to microwave links. In contrast to universal monitoring systems, it contains integrated methods of analysis, detecting sudden and long-term changes in parameters. The indication of total outages is included as a basic standard. By implementing other methods such as the above-mentioned temperature correlation and connecting basic methods with the evaluation of the atmospheric situation, the application can become a useful tool for NOC operators.

\section{ACKNOWLEDGEMENT}

This project was supported by company CBL Communication by light s.r.o. which provided the test range of real microwave units hardware with common traffic load for the purposes of this work.

\section{REFERENCES}

[1] KIZER, George. Digital Microwave Communication: Engineering Point-to-Point Microwave Systems. New Jersey: Wiley-IEEE Press, 2013. ISBN 978-0-470-12534-2.

[2] LEHPAMER, Harvey. Transmission Systems Design Handbook for Wireless Networks. Norwood: Artech House, 2002. ISBN 1-58053-243-8.

[3] HILES, Andrew. E-Business Service Level Agreements. Brookfield, CT: Rothstein Associates, 2002. ISBN 0-9641648-9-2.

[4] RUDOLPH, Kevin. A Comparison of NoSQL Time Series Databases. Berlin: GRIN Publishing, 2015. ISBN 978-3656965763.

[5] ALBAHARI, Joseph a Ben ALBAHARI. C\# 7.0 in a nutshell. 7th edition. Sebastopol, CA: O'Reilly, 2018, 1070 s. ISBN 978-1-491-98765-0. 